\title{
Molecular Docking Simulation Based Virtual Screening for The Design of Potential Inhibitors of Heme Oxygenase of Corney Bacterium Diphtheria.
}

\author{
Somdutt Mujwar, Raghav Mishra, Isha Tomer, Alekh Gour
}

\begin{abstract}
Diphtheria is an infectious human disorder affecting upper respiratory tract which is characterized by fever, sore throat, and malaise. It is caused by Corynebacterium diphtheria and other pathogenic strains of Corynebacterium. The pathogen invades in the nasopharynx and infects the host by releasing an exotoxin leading to the severe concerns in thekidneys, nervous system and cardiac muscles. Sometimes diphtheria infections may be fatal because of the circulatory failure caused by myocarditis.Hemeoxygenase is the rate limiting enzyme in heme degradation and catalyzes the NADPHcytochrome $P 450$ reductase-dependent cleavage of heme to biliverdin with the release of iron and carbon monoxide. In the present paper we have performed molecular docking simulation based in-silico virtual screening of an NCI diversity set-II containing 1593 diverse ligands to identify potential inhibitor of the Heme oxidase enzyme of Corneybacterium diphtheria. The lead molecules are shortlisted on the basis of their binding energy and these molecules are supposed to be further evaluated experimentally for development of a newer therapy for the treatment of diphtheria.
\end{abstract}

Keywords: Corney bacterium diphtheria; Diphtheria; Heme oxygenase: Molecular docking: virtual screening.

\section{INTRODUCTION}

During the recent past, there is a rise in the human population which are getting affected by life-treating infectious diseases. Microbial drug resistance hasn't simply supplemented in the expansion in the rate of mortality identified with infections and the frequency of diseases, stillthere isbudgetary increasein the health care expenses for the treatment for drug-resistant infections [1]. On account of the dramatic escalation in microbial infections, newer antimicrobial agents havingunique mechanisms are earnest need to fight against the multidrug-resistant infections.

The medicinal chemists are primarily exercisingnowadays on the discovery and development of new biologically active agents for the treatment of these alleviating ailments.

Revised Manuscript Received on 30 July 2019.

* Correspondence Author

(Dr. SomduttMujwar)*, Assistant Professor, Institute of Pharmaceutical Research, GLA University, Mathura-281406, Uttar Pradesh, India.

Mr. Raghav Mishra, Assistant Professor, Institute of Pharmaceutical Research, GLA University, Mathura-281406, Uttar Pradesh, India.

Mrs. Isha Tomer, Assistant Professor, Institute of Pharmaceutical Research, GLA University, Mathura-281406, Uttar Pradesh, India.

Dr. Alekh Gour, Assistant Professor, Department of Biological Management, Goa Institute of Management, Sanquelim-403505, Goa, India

(C) The Authors. Published by Blue Eyes Intelligence Engineering and Sciences Publication (BEIESP). This is an open access article under the CC-BY-NC-ND license http://creativecommons.org/licenses/by-nc-nd/4.0/
The design and development of drug molecules clearly bidsundoubtedlywith the utmost hopes for endeavors in the present and future era [2].

Diphtheria is an uncommon disease in most developed nations inferable from the routine childhood vaccination. However, the disease is seen at times among nonimmunized children in developing countries. [3] Diphtheria is brought about by a toxigenic bacterium Corynebacteriumdiphtheriae which stays as one of the imperative reasons of illness and death among children. There are four principle biotypes of $C$. diphtheriae: Gravis, Intermedius, Mitis, and Belfanti. Globally, the Intermedius is most often associated with exotoxin production, despite all three strains are able of producing exotoxin. [4] It is an overwhelming life threatening situation, with the issues requiringtimely recognition, fast treatment against the diphtheria antitoxin and in the most extreme circumstancescritical intensive care interventions to comfort the obstructions of the upper respiratory system. Diphtheria toxin causes quick obstruction of the upper airway by the massive soft tissue oedema, acute and subacute cardiac, the formation of the pseudo- membrane in the pharynx, larynx and nares, acute and subacute cardiac, renal and neurological complications. In spite of the availability of the diphtheria vaccine since 1921, its appropriation was limited in the initial days and a million diphtheria cases were reported and over 60,000 deaths were happened every year in the 1970s [5]. The incorporation of diphtheria toxoid vaccine in the worldwide Expanded Programme on Immunization in 1974 saw a decrease of cases by over $90 \%$ from 1980 to 2000. With a fundamental proliferation number of 6 to 7 (average number of secondary cases in a fully susceptible population), only $85 \%$ of each birth associate need to be immunized to achieve the critical immunity threshold that gives diphtheria herd immunity. [6] Three dosage of the vaccine containingdiphtheria toxoid has been continued universally since 2010, despite the fact that with the increasing country to country variations as well as the rise of re-centre proves the homogenous coverage of population by the diphtheria. The systemic indications of diphtheria are because of serious tissue damage throughout the body caused by the excretion of the diphtheria exotoxin [7]. The diphtheria toxin wasencoded by the tox gene, which has been appeared to be under transcriptional control of the iron-dependent diphtheria toxin repressor protein [8]. It is a global iron-dependent repressor likened to the ferric uptake repressor protein of Gram-negative bacteria.

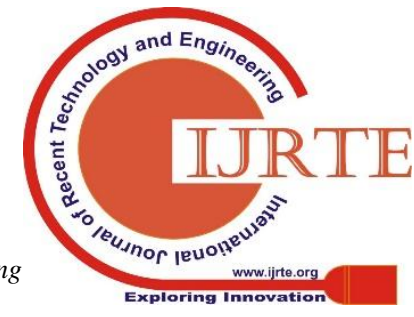




\section{Molecular Docking Simulation Based Virtual Screening for The Design of Potential Inhibitors of Heme Oxygenase of Corney Bacterium Diphtheria.}

Iron is a basic supplement required for the survival of most bacteria, and the capacity of pathogenic bacteria to get adequate iron during infection is essential for such pathogens to cause disease [9]. To dodge the low availability of iron, pathogens have developed refined mechanisms to utilize the host's iron- and heme-containing proteins. Hemeoxygenase is the rate limiting enzyme in heme degradation and catalyzes the NADPH-cytochrome P450 reductase-dependent cleavage of heme to biliverdin with the release of iron and carbon monoxide. [10] In the present paper we have performed molecular docking simulation based in-silico virtual screening of an NCI diversity set-II containing 1593 diverse ligands to identify potential inhibitor of the Heme oxidase enzyme of Corneybacterium diphtheria. The Heme oxidase inhibitors are supposed to interfere with the life cycle of the pathogen and should act as a potential therapy for the treatment of diphtheria.

\section{EXPERIMENTAL}

\section{A. Selection of Macromolecule and its Preparation}

The Hemeoxygenase $(\mathrm{HeO})$ enzyme of Corneybacterium diphtheriabound with ligand protoporphyrin-IX (HEM) (pdb id-1v8x) was downloaded from the protein data bank (Figure.1). The Hemeoxygenase enzyme was prepared for molecular docking simulation by removing ligand and from its active binding site, removal of water molecules which are not interacting with the ligand, and addition of polar hydrogen atoms [11-12].

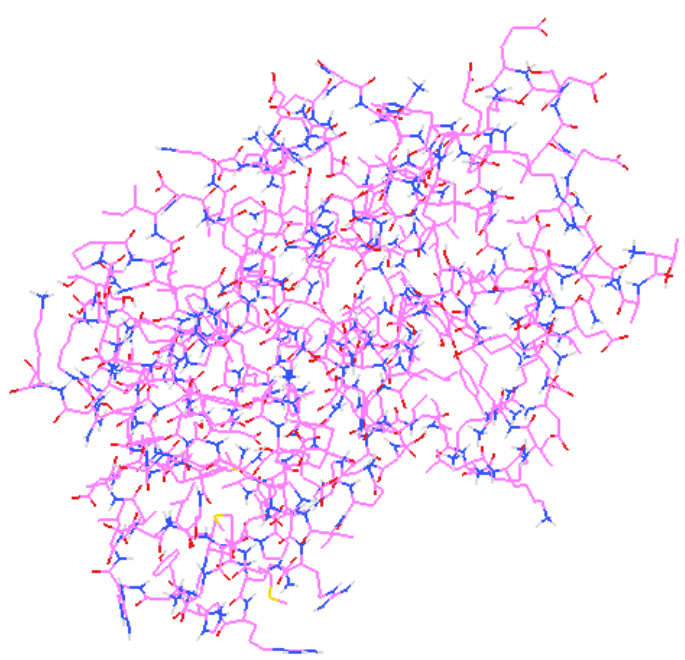

Figure 1. Crystal structure of the Hemeoxygenase enzyme of $C$. diptheria acquired from the protein data bank database. (PDB ID-1v8x)

\section{B. Preparation of Ligand for Molecular Docking}

The bound ligand HEM is prepared for molecular docking simulation by providing the rotatable, non-rotatable as well as un-rotatable bonds present in the ligand to the AutoDock software [3].

\section{Identification of Binding Site}

The ligand binding site of the HemeOxygenase enzyme is identified by exploring the binding interaction of the bound ligand HEM by using PyMol software. The complexed ligand HEM bound in the receptor's active binding site is separated from the complex molecule by using software chimera [14-15].

\section{Molecular Docking}

The binding site of the Hemeoxygenase is identified by using protein visualization PyMolsoftware to enumerate the grid parameter points of the grid box required to perform the molecular docking simulation of ligand molecules with pathogenic Hemeoxygenase. These grid parameters were utilized for all docking runs. The grid-box was placed by centering the ligand molecule and covering all the residues involved in the binding of the ligand to ensure that all the extended conformations of ligand fits within the grid box (Figure 2).

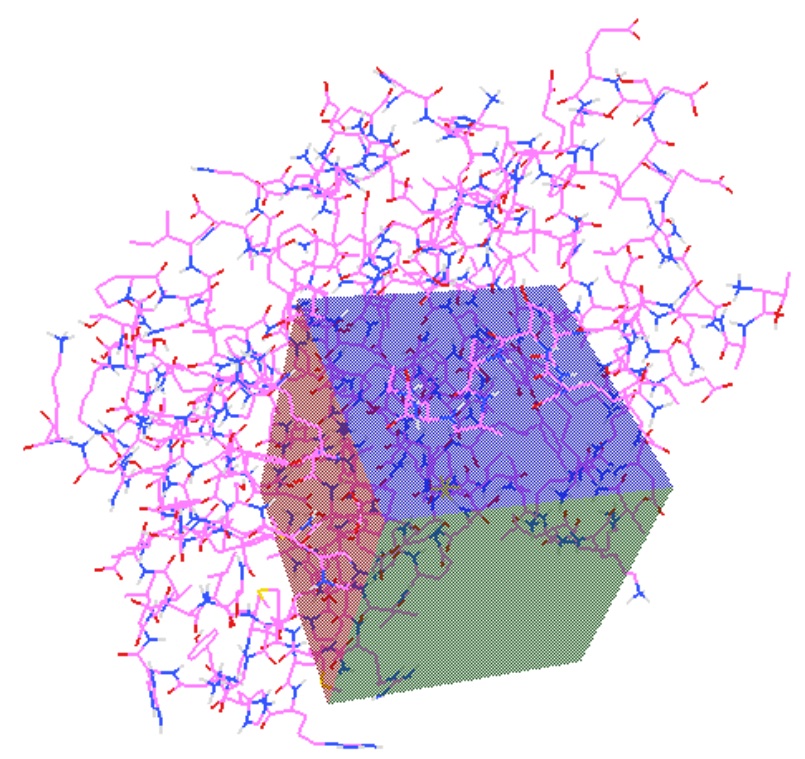

Figure 2. Three-dimensional grid-box covering the ligand binding site of the pathogenic Heme oxygenase enzyme.

The separate map files for each of the atom types present in the receptor as well as ligand viz. A Cl OA SA F H P Br Fe HD N C I NA $S$ etc. are prepared by running Autogrid utility of the AutoDock suite. These map file prepared by Autogrid is used for carrying out molecular docking simulations by AutoDock [16-17]. Lamarckian genetic algorithm (LGA) is one of the primary conformational search approach employed in AutoDock for molecular docking simulation. A trail population is created for various possible conformations, followed by the mutation, conformational parameters exchange, and compete in a manner kindred to biological evolution in successive generations for eventually selecting individuals with lowest binding energy. The individual conformational search for its local conformational space, discovering local minima, and then proceed this information to later generations is performed by "Lamarckian" aspect, which is its additional feature. 
The binding energy of the small molecules with macromolecular targets is predicted by using semi-empirical force field. The force field allows the assimilation of the intramolecular energies into the predicted binding energy by the evaluation of the energetics for both bound as well as unbound states based on a comprehensive thermodynamic model. Docking parameter file required for the docking of each ligand molecule was prepared by using the 150 Genetic Algorithm (GA) runs, 250000 maximum numbers of evaluations, 27000 maximum numbers of generations and $0.02 \%$ rate of gene mutation [16-17].

\section{E. Docking Method Validation}

The position and orientations of the ligand obtained after the molecular docking study represents probable binding patterns of the inhibitors. The various docking parameters considered in the docking methods were validated by redocking individually crystallized ligand HEM over Hemeoxygenase enzyme. The molecular docking simulation technique is validated by using following parameters:

\section{(a) Overlay Methods}

The validation of molecular docking method is performed by overlay method. The docked conformation of the bound ligand should be impeccably overlaid with reference to the bioactive conformation of the ligand present in the crystal structure of the downloaded protein.

\section{(b) Chemical Resemblance}

The molecular docking method is validated when the docked ligand should have same interactions with the residues of macromolecule as that present in the downloaded crystallized macromolecule.

\section{F. In-silico Virtual Screening}

In the present studythe NCI diversity set-II containing 1593 diverse ligand molecules were virtually screened against validated pathogenic Hemeoxygenase enzyme. The molecular docking simulation based in silicovirtual screening is performed to identify potential inhibitor molecules for Hemeoxygenease enzyme of $C$. diphtheria.

\section{G. Analysis of Molecular Docking Simulation Results}

After performing molecular docking simulation of the selected ligand molecules against the pathogenic Hemeoxygenase enzyme, the best ligand molecule were evaluated on the basis of their lowest binding energy. The Lamarckian Genetic Algorithm used for scoring. All the results obtained by molecular docking simulation were evaluated on the basis of hydrophilic and lipophilic interactions obtained between the binding residues present in the active ligand binding site of the macromolecule and ligand. The empirical range of the free binding energy should be in the range of -5 to $-15 \mathrm{kcal} / \mathrm{mol}$. The mathematical equation to calculate binding affinity of the specific ligand for a particular target is as follows:

$$
\mathbf{K}_{\mathbf{i}}=\mathbf{e}^{[(\Delta \mathrm{G} /(\mathrm{RT})]}
$$

Where, $\Delta \mathrm{G}=$ change in free energy upon binding, $\mathrm{R}=$ gas constant and $\mathrm{T}=$ temperature.

\section{H. Pharmacokinetic and Toxicity Evaluation}

The physicochemical parameters as well as toxicity profile of all the selected lead molecules were evaluated by using DataWarrior software. DataWarrior software checks for the presence of major toxic effects such as mutagenicity, tumorigenecity, irritant effect and reproductive effects in the lead molecules. This software searches for the presence of those functional group which are responsible for the toxic effects of the ligand molecule. The DataWarrior program also calculates drug-likeness and drug score of the lead molecule on the basis of their physicochemical properties [17].

\section{RESULTS AND DISCUSSION}

\section{A. Selection and Preparation of Macromolecule}

Hemeoxygenase bound withligand HEM (pdb id-1v8x) was downloaded from protein data bank database. The three dimensional structure model of protein is procured by using $\mathrm{X}$-ray differaction technique at a resolution of $1.85 \AA$ by using E. coli as an expression system. The $1 \mathrm{v} 8 \mathrm{x}$ protein complex consists of three identical polypeptide chain of 215 amino acids. The chain $B$ and $C$ were removed with the help of chimera software and chain A was selected for the experiment. The receptor molecule is prepared for molecular docking simulation process by adding polar hydrogen bonds, removing redundant water molecules and addition and distribution of charge. After processing the receptor molecule it was saved in *.pdbqt format by using AutoDock software.

\section{B. Preparation of Ligand for Molecular Docking}

Eight rotatable bonds were present in the bound ligand molecule. All the eight bonds were kept rotatable in the ligand molecule for the current experimental study. The prepared ligand was saved in the *.pdbqt format.

\section{Identification of Binding Site and Grid-Box Preparation}

The amino acid residues Tyr130 and Arg177 were involved in the active binding of the HEM ligand with the pathogenic Hemeoxygenase enzyme. The binding interactions of the bound ligand HEM ligand with the pathogenic Hemeoxygenase enzyme is shown in Figure 3.

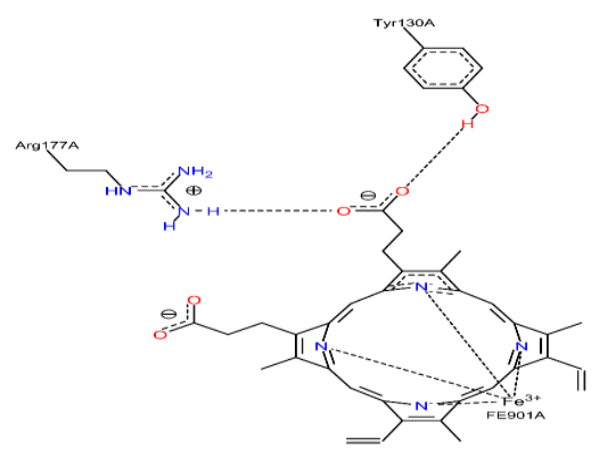

Figure 3. The binding interactions of the bound ligand HEM ligand with the pathogenic Heme oxygenase enzyme. 
Molecular Docking Simulation Based Virtual Screening for The Design of Potential Inhibitors of Heme Oxygenase of Corney Bacterium Diphtheria.

An appropriate grid box was prepared by covering all the macromolecular residues which are involved in the active binding of the bound ligand HEM ligand with the pathogenic Hemeoxygenase enzyme. The coordinates used for the preparation of the grid box were tabulated in table 1.

Table 1. The grid coordinates for pathogenic Heme oxygenase enzyme.

\begin{tabular}{|l|l|l|l|l|l|l|l|}
\hline Proteins & $\begin{array}{l}\mathrm{x}- \\
\mathrm{D}\end{array}$ & $\begin{array}{l}\mathrm{y}- \\
\mathrm{D}\end{array}$ & $\begin{array}{l}\mathrm{z}- \\
\mathrm{D}\end{array}$ & $\begin{array}{l}\text { Spacing } \\
(\mathrm{A})\end{array}$ & $\begin{array}{l}\mathrm{x} \\
\text { center }\end{array}$ & $\begin{array}{l}\mathrm{y} \\
\text { center }\end{array}$ & $\begin{array}{l}\mathrm{z} \\
\text { center }\end{array}$ \\
\hline 1v8x & 40 & 40 & 40 & 0.425 & 25.134 & -9.27 & 1.154 \\
\hline
\end{tabular}

D. Molecular Docking Simulations and Its Validation

The results obtained after molecular docking of the bound ligand HEM ligand with the pathogenic Hemeoxygenase enzyme were presented in table 2.

Table 2. Molecular Docking results of ligand HEM ligand with the pathogenic Heme oxygenase enzyme (1v8x).

\begin{tabular}{|c|c|c|c|}
\hline Protein & $\begin{array}{c}\text { Interacting } \\
\text { residue }\end{array}$ & RMSD & $\begin{array}{c}\text { Binding energy } \\
\text { (kcal/mol) }\end{array}$ \\
\hline 1v8x & $\begin{array}{c}\text { Tyr130 and } \\
\text { Arg177 }\end{array}$ & 0.41 & -11.92 \\
\hline
\end{tabular}

The molecular docking process for docking of particular ligand with a specific macromolecule was performed by considering following parameters:-

\section{(a) Overlay Method}

The molecular docking method was validated as the docked conformation of the ligand should be perfectly overlaid with the crystal structure of the ligand present in the downloaded protein. The overlaid conformation of the docked ligand with reference to the crystal structure of the downloaded ligand was shown in figure 4.

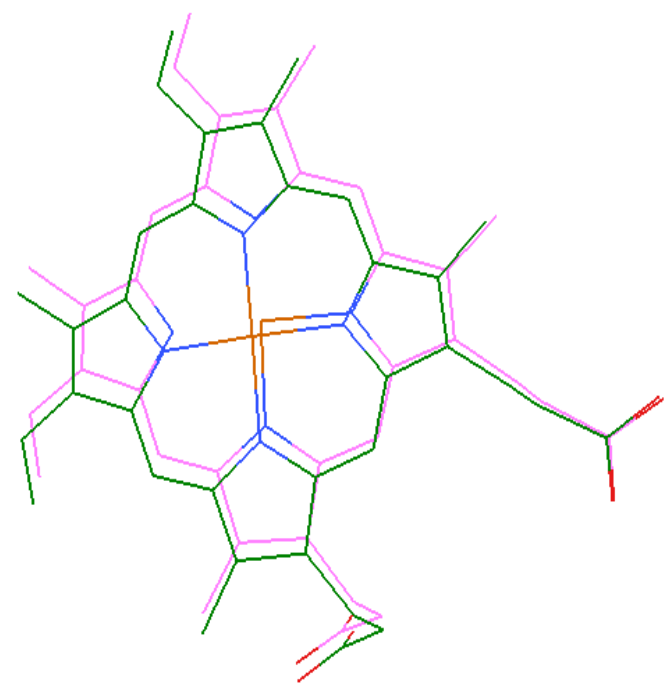

Figure 4. The superimposition of the docked conformation of the ligand with reference to its bioactive conformation of the ligand obtained from the crystal structure of the downloaded protein.

\section{(b) Chemical Resemblance}

The molecular docking method was validated when the docked ligand had the similar interactions with the residues of macromolecule as that were present in the downloaded crystallized macromolecule. The interactions present in crystal structure and the interactions present in the docked structure were shown in figure 5 .
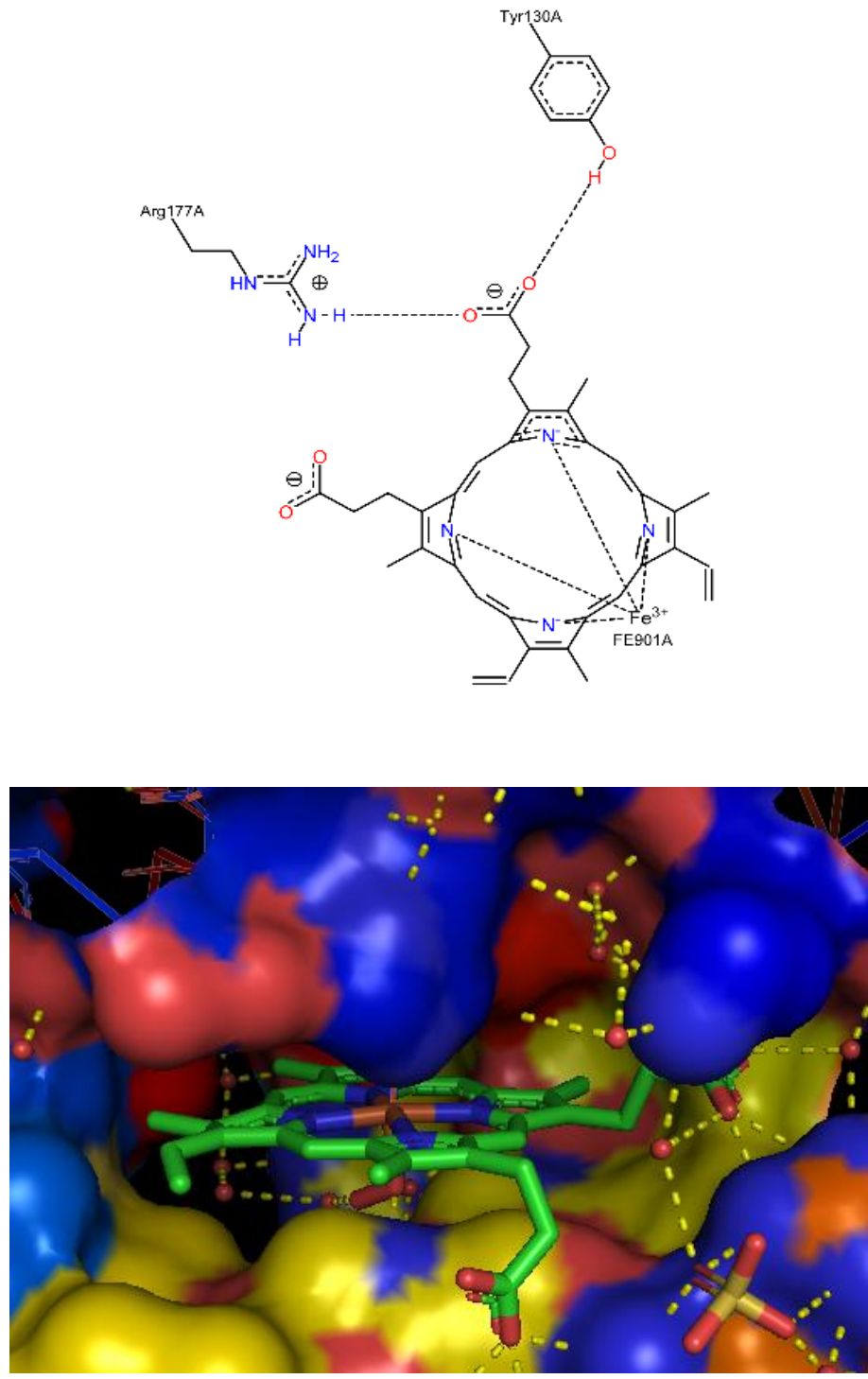

Figure 5. Binding mode and chemical interactions of the bound ligand HEM within the active ligand binding site of pathogenic Heme oxygenase enzyme.

\section{E. Virtual Screening}

The binding affinity of all the ligand molecules were identified by analyzing the binding energy obtained for top ranking pose of each ligand and interactions of docked compound were visualized. The molecular docking results of all the nine flavonoid molecules were obtained after performing AutoDock based molecular docking simulation against the pathogenic Hemeoxygenaseenzyme were shown in table 3 .

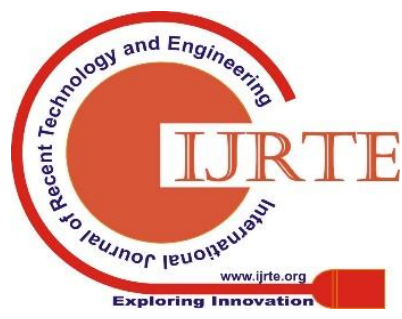


Table 3. Binding energy of selected best ligand molecules against the pathogenic Hemeoxygenase enzyme.

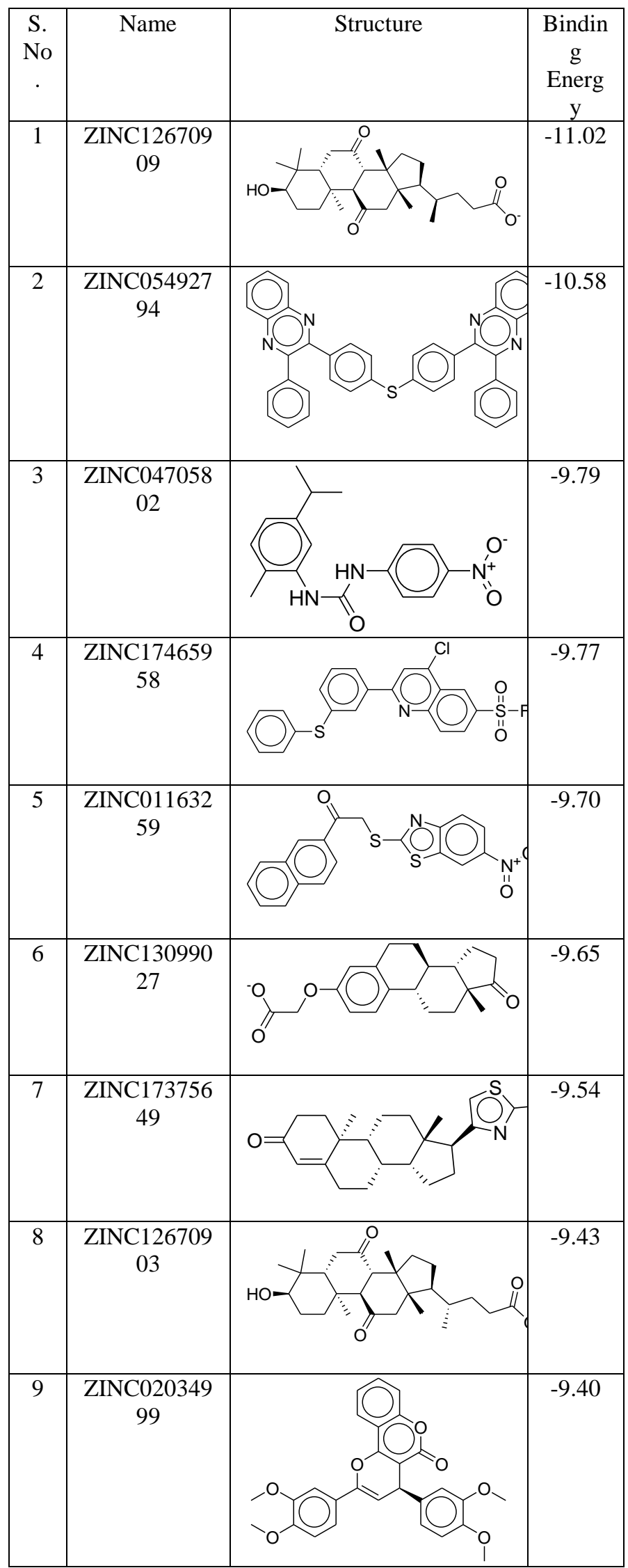

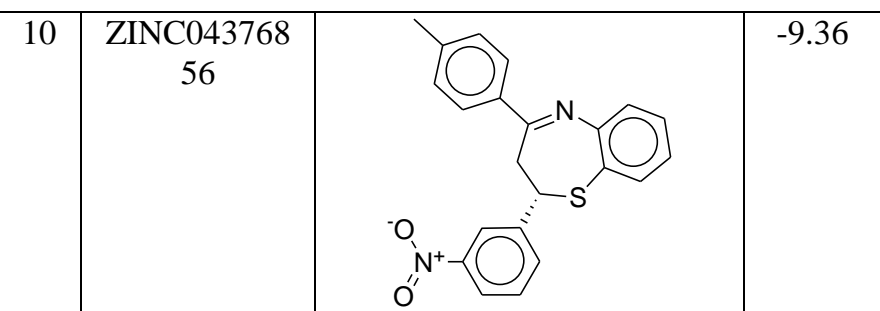

\section{F. Pharmacokinetic and Toxicity Evaluation}

The toxicity evaluation of the lead molecules by observing the presence of functional groups responsible for the major toxic effects like mutagenicity, tumorogenicity, irritant effect as well as reproductive effect were identified by using DataWarrior software. The results obtained in the toxicity profiling of all the selected lead molecules are shown in the table 4 . The results of toxicity profiling of all lead molecules suggests that the five out of ten compound, i.e. ZINC12670909, ZINC01163259, ZINC12670903, ZINC02034999 and ZINC04376856 are not having any functional group responsible for major toxic effects like mutagenicity, tumorogenicity, irritant effect as well as reproductive effect with very good pharmacokinetic profile.

Table 4: Toxicity profiling for the pathogenic Heme oxidase inhibitors.

\begin{tabular}{|l|l|l|l|c|}
\hline Lead Compound & Mutagenic & Tumorigenic & Irritant & $\begin{array}{c}\text { Reprodu } \\
\text { ctive } \\
\text { Effect }\end{array}$ \\
\hline ZINC12670909 & None & None & None & None \\
\hline ZINC05492794 & High & None & None & High \\
\hline ZINC04705802 & None & None & High & High \\
\hline ZINC17465958 & None & None & High & High \\
\hline ZINC01163259 & None & None & None & None \\
\hline ZINC13099027 & None & None & None & High \\
\hline ZINC17375649 & None & None & None & High \\
\hline ZINC12670903 & None & None & None & None \\
\hline ZINC02034999 & None & None & None & None \\
\hline ZINC04376856 & None & None & None & None \\
\hline
\end{tabular}

\section{CONCLUSION}

AutoDock based molecular docking simulation technique is very useful in identification of mechanism of action for some of the potential inhibitors for Heme oxidase enzyme of C. diphtheriae. The NCI diversity set-II containing 1593 diverse ligands are evaluated for their binding affinity against the pathogenic Heme oxidase enzyme.The ligand molecules having best binding affinity are identified as a lead molecules having potential inhibitory activity of pathogenic Heme oxidase enzyme. These selected lead molecules are further evaluated for their pharmacokinetic profiling and presence of toxic effects. The results of toxicity profiling of all lead molecules suggests that the five out of ten compound, i.e. ZINC12670909, ZINC01163259, ZINC12670903, ZINC02034999 and ZINC04376856 are not having any functional group responsible for major toxic effects like mutagenicity, tumorogenicity, irritant effect as well as reproductive effect with very good pharmacokinetic profile. Thus, these five molecules are supposed to be further evaluated experimentally for development of a newer therapy for the treatment of diphtheria. 


\section{Molecular Docking Simulation Based Virtual Screening for The Design of Potential Inhibitors of Heme Oxygenase of Corney Bacterium Diphtheria.}

\section{REFERENCES}

1. R. C. MoelleringJr,"Discovering new antimicrobial agents", Int $J$ Antimicrob Agents, Vol.37, No.1, pp.2-9, 2011.

2. R. Mishra,P. K. Sharma, P. K.Verma,I.Tomer, G. Mathur, P. K. Dhakad,"Biological potential of thiazolederivatives of synthetic origin."J HeterocyclChem, Vol.54, No. 4, pp.2013-16, 2017.

3. World Health Organisation. Expanded program on immunization (EPI) information system. Geneva: WHO; 1993.

4. T. Ramamurthy,S. Azim, S.Ganguly, S. K. Bhattacharya, “'Diphtheria: An Emerging Disease."J Clin Infect Dis, Vol.3 No.1, pp.121-23, 2018.

5. T. S. P. Tiwari, M. Wharton,"Diphtheria toxoid. Plotkin's vaccines" $7^{\text {th }}$ edition, Elsevier, pp.261-75, 2017.

6. R. M. Anderson, R. M. May, Infectious diseases of humans. Dynamics and control. Oxford: Oxford University Press; Vol.70, 1992.

7. A. B. Christie,"Infectious Disease: Epidemiology and Clinical Practice", Churchill Livingstone, London, pp. 1183-09, 1987.

8. M. P. Schmitt, R. K. Holmes, "Iron-dependent regulation of diphtheria toxin and siderophore expression by the cloned Corynebacteriumdiphtheriae repressor gene dtxR in C. diphtheriae C7 strains",Infect Immun, Vol.59, No.6, pp.1899-904, 1991.

9. E. D. Weinberg, "Iron and infection",Microbiol Rev, Vol.42, No. 1, pp.45, 1978.

10. B. A.Schacter, E. B. Nelson, H. S. Marver, B. S. Masters, Immunochemical evidence for an association of hemeoxygenase with the microsomal electron transport system. J BiolChem,Vol 247, No. 11, pp.3601-07, 1972.

11. H. M. Berman, J. Westbrook, Z. Feng, G. Gilliland, T. N. Bhat, H. Weissig, I. N. Shindyalov, P. E. Bourne,"The protein data bank."Nuc. Acid Res, Vol.28. No.1, pp.235-42, 2000.

12. M. C.Franklin, M. J. Rudolph, C. Ginter, M. S. Cassidy, J. Cheung,"Structures of paraoxon-inhibited human acetylcholinesterase reveal perturbations of the acyl loop and the dimer interface",Proteins, Vol. 84, No.9, pp.1246-56, 2016.

13. Goodsell DS, Morris GM, Olson AJ. Automated docking of flexible ligands: applications of AutoDock. Journal of Molecular Recognition. 1996 Jan;9(1):1-5.

14. W. L.DeLano,"Pymol: An open-source molecular graphics tool", CCP4 Newsletter On Protein Crystallography. Vol.40, No.1, pp.82-92, 2002.

15. E. F. Pettersen, T. D. Goddard, C. C. Huang, G. S. Couch, D. M. Greenblatt, E. C. Meng, T. E. Ferrin, "UCSF Chimera-a visualization system for exploratory research and analysis",JComputChem,, Vol.25, No.13, pp.1605-12, 2004

16. S. Mujwar, K. R. Pardasani,"Prediction of Riboswitch as a potential drug target for infectious diseases: An Insilico case study of anthrax."J Med Imaging Health Inform, Vol.5, No.1, pp.7-16, 2015.

17. T. Sander, J. Freyss, M. von Korff, C. Rufener,"DataWarrior: an opensource program for chemistry aware data visualization and analysis",JChem Inform Model, Vol55. No.2, pp.460-73, 2015.

18. S. Mujwar, K. R. Pardasani,"Prediction of riboswitch as a potential drug target and design of its optimal inhibitors for Mycobacterium tuberculosis",Int J ComputBiol Drug Des. Vol.8, No.4, pp.326-47, 2015.

\section{AUTHOR PROFILE}

Dr. Somdutt Mujwar I'm a researcher working in the field of bioinformatics and computational biology having sound knowledge of Pharmaceutical Chemistry. My research interests revolves aroundunderstanding the pathophysiology of various diseases and using knowledge acquired frommy pharmaceutical background and its application in my current research studies for designof novel lead molecules with high potency and free from side effects as well as developmentof drug resistance by the pathogenic microorganisms. Presently I'm working on the In-silico design of riboswitch based novel inhibitor molecules for various pathogenic microorganisms which can be free from associated side effects aswell as development drug resistance by the pathogen. I am using AutoDock based virtualscreening technique to screen potent lead molecules, which are further used for thedevelopment of pharmacophore. This designed pharmacophore structure is used for thedevelopment of novel inhibitor molecules by using bioisosteric substitution technique, whichare further evaluated for their pharmacokinetic properties on the basis of theirphysicochemical properties.

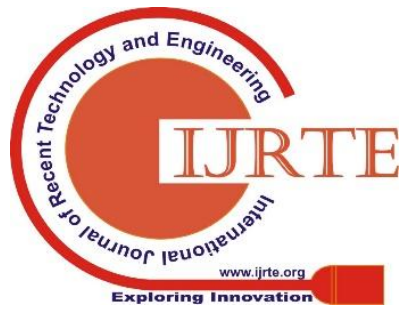

